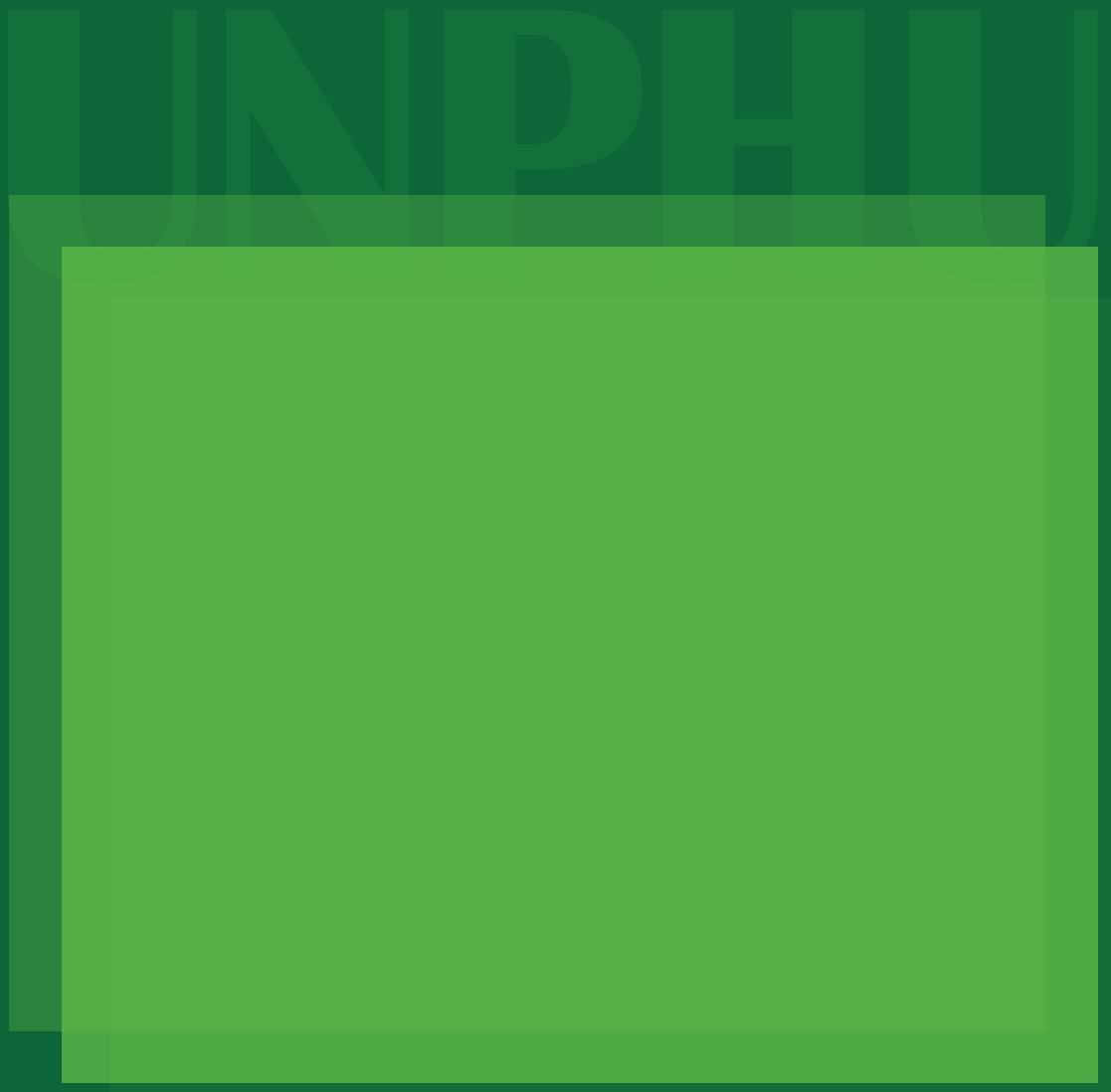




\title{
Gestión de Riesgos (Sendai) y Objetivos Desarrollo Sostenible en Puerto Plata
}

\author{
Juan Roberto Suriel Guzmán \\ Director del Departamento de Medio Ambiente y Recursos Naturales, UNPHU. \\ Santo Domingo, República Dominicana \\ r.suriel@unphu.edu.do
}

Fecha de recepción: 9 de enero de 2018

Fecha de aceptación: 9 de abril de 2018

Favor de citar este artículo de la siguiente forma:

Suriel Guzmán, J. (2018). Gestión de Riesgos (Sendai) y Objetivos Desarrollo Sostenibles en Puerto Plata.

Revista AULA. Vol.62, Número 1,enero-junio 2018. Santo Domingo : Amigo del Hogar.

\section{RESUMEN}

Este artículo trata sobre los desastres recurrentes del Municipio de San Felipe de Puerto Plata y cómo el Acuerdo Marco para la Reducción de Desastres 2015-2030 de Sendai, junto a los Objetivos de Desarrollo Sostenible (ODS), se interrelacionan y de cómo pueden ser utilizados para la Gestión de riesgos ante desastres. En él se muestran trabajos previos sobre gestión de riesgos de desastres realizados en este territorio. Esto es importante porque parece que no han sido comprendidos en toda su extensión por las autoridades y las comunidades. Prueba de ello son los efectos negativos, repetitivos, de amenazas naturales y antrópicas convertidas en realidad como ocurrió con las pérdidas ocurridas durante las lluvias de los meses de octubre y noviembre de 2016, las vaguadas de inicio del año 2017 y las ocasionadas durante el paso por las cercanías de República Dominicana de los huracanes Irma y María en el mes de septiembre de 2017. Los hallazgos encontrados mostraron que existe insuficiencia de entendimiento y sensibilidad de parte de los actores claves del Municipio de San Felipe de Puerto Plata al trabajo coordinado de la gestión de riesgos a desastres naturales y antrópicos. Se recomienda desarrollar proyectos a nivel local, del gobierno central y organismos internacionales tomando en cuenta el Marco de Sendai y los Objetivos de Desarrollo Sostenible (ODS) y la Estrategia Nacional de Desarrollo, de manera que los trabajos, además de aumentar la sostenibilidad ambiental, logren el futuro que queremos, aumente la resiliencia y se reflejen en los indicadores de ODS y las metas e indicadores del Marco de Sendai.

Palabras clave: cambio climático, gestión de riesgos, objetivos de desarrollo sostenible Puerto Plata, Sendai 


\begin{abstract}
This article refers to the recurring disasters affecting the Municipality of San Felipe de Puerto Plata and how the Framework Agreement for Disaster Reduction 2015-2030 of Sendai, together with the Sustainable Development Goals (SDG), are interrelated and can be used for a Disaster Risk Management. It shows previous work on disaster risk management in this territory. This is important for it seems it have been poorly understood by authorities and communities. Prove of this, is the repetitive negative effects of natural threats and anthropic occurrences; as it occurred with the losses during the rains of October and November 2016, the beginning of 2017, and those caused during the passage through the vicinity of the Dominican Republic of Hurricanes Irma and María in September 2017. The findings showed that there is insufficient understanding and sensitivity, from the key stakeholders of the Municipality of San Felipe de Puerto Plata, to the coordinated work of risk management of natural and anthropic disasters. The development of projects at the local level by the central government and international organizations, taking into account the Sendai Framework, the Sustainable Development Goals and the National Development Strategy. The recommended works, in addition to increasing Environmental Sustainability, would achieve the future we want, increase resilience and reflect it in the SDG indicators of the Sendai framework.
\end{abstract}

Palabras clave: climate change, risk management, Sustainable Development Goals (SDG), Puerto Plata, Sendai

\title{
Gestión de Riesgos (Sendai) y Objetivos Desarrollo Sostenible en Puerto Plata
}

\section{Introducción}

Los resultados recurrentes de inundaciones asociadas a las precipitaciones del mes de noviembre de 2016, las vaguadas del inicio del año 2017 y las ocasionadas durante el paso por las cercanías de República Dominicana (RD), de los huracanes Irma y María en el mes de septiembre de 2017, llevaron a las autoridades a declarar la provincia de Puerto Plata con todos sus municipios en estado de emergencia en cada uno de estos fenómenos naturales.

El centro de Operaciones de Emergencias (COE), en el "Informe final por vaguada del 8 de diciembre de 2016" (COE Vaguada, 2017), el "Informe final del huracán Irma del 15 de septiembre de 2017" (COE, 2017) y el "Informe final del huracán María del 02 de octubre de
2017” (COE, 2017), así como visitas al área de estudio, muestran cómo Puerto Plata fue afectada por estos fenómenos naturales.

La motivación de escribir este artículo se origina a partir mi experiencia como director del "Proyecto para el Fortalecimiento de las Estructuras Organizativo-Funcionales de la Gestión de Riesgos Ante Desastres en la RD”, de la Presidencia de la República Dominicana y la Unión Europea durante el año 2015. Esto me permitió conocer la situación presente en muchos municipios del país; que afecta el desarrollo de los mismos mientras no se intervenga adecuadamente. Por mi interés de cooperar con el país, una motivación más, pude observar bien de cerca el sufrimiento de esos dominicanos afectados por los desastres que necesitan de un trabajo como nación en beneficio de todos. Además, este articulo incluye parte de 
las conclusiones del proyecto de investigación que realicé en el curso "Reducción de Riesgo de Desastres y Desarrollo Local Sostenible", del Centro Internacional de Formación de la Organización Internacional del Trabajo (OIT) y la oficina de la Naciones Unidas (UN) para la Reducción de Riesgos de Desastres (UNISDR) en Turín, Italia.

La República Dominicana es miembro de Naciones Unidas, que el día 25 de septiembre de 2015 adoptó de conjunto, 17 Objetivos de Desarrollo Sostenible. También, la R.D. firmó el 18 de marzo de 2015 un Acuerdo sobre Gestión de Riesgos a Desastres, el Marco de Sendai, que es el instrumento sucesor del Marco de Acción de Hyogo, con un principio fundamental de aumento de la resiliencia de las naciones y las comunidades ante los desastres.

Este artículo se enmarca en la aplicación del Acuerdo Marco para la Reducción de Desastres 2015-2030 de Sendai y los Objetivos de Desarrollo Sostenible (ODS) en el Municipio de San Felipe de Puerto Plata.

\section{Desarrollo}

En el Informe de la vaguada del 8 de diciembre de 2016 del COE (COE Vaguada, 2017), Puerto Plata encabezó la lista de comunidades afectadas con cierre de vías por deslizamientos de tierra, crecidas de ríos, cañadas, afectaciones de puentes y pérdidas de vidas humanas. Durante la visita realizada el 26 de noviembre de 2016 al área del Municipio de San Felipe de Puerto Plata, el Director de la Defensa Civil, mediante comunicación oral, reportó que en las precipitaciones de ese mes resultaron unas 5,714 casas inundadas, 28 casas destruidas, junto a una cantidad de desplazados de 28,710 personas. En las fotos se muestra el desarrollo de la investigación y daños a vivienda producida por la erosión provocada por el río San Marcos.

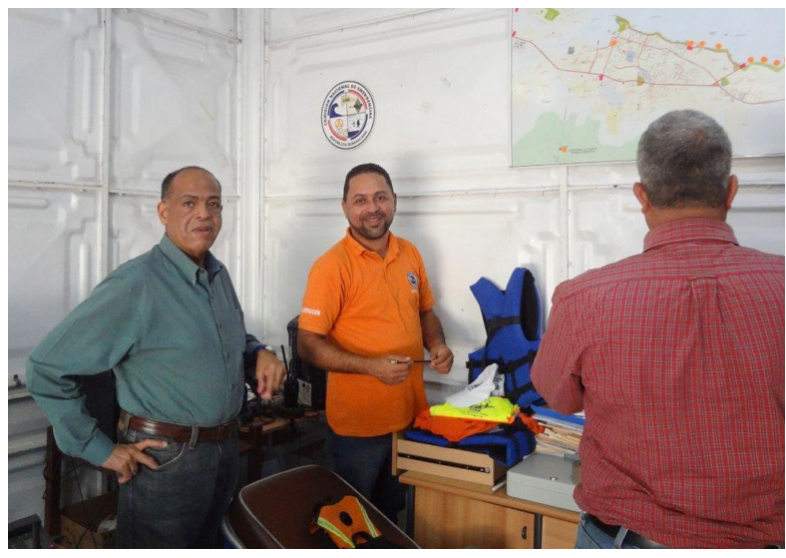

Imagen I. Reunión con el director de la Defensa Civil de Puerto Plata

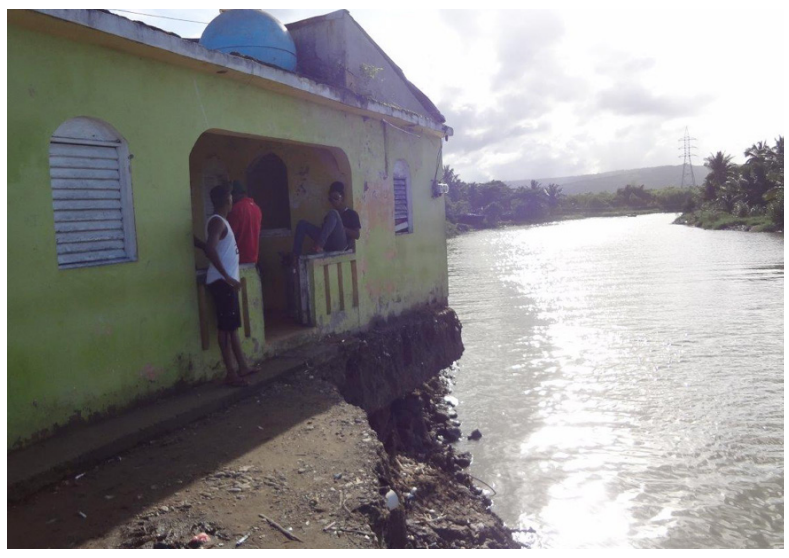

Imagen II. Erosión cimientos vivienda provocada por el río San Marcos

En el Informe del Huracán Irma del 15 de septiembre de 2017 del COE, se plasma que Puerto Plata estuvo de nuevo dentro de los municipios más afectados con personas movilizadas, viviendas afectadas y destruidas, además de infraestructura vial con daños parciales o totales, servicios básicos afectados, pérdidas de medios de vida, daños severos a la agropecuaria y un muelle afectado (COE Irma, 2017).

Con el huracán María del 02 de octubre de 2017, el COE reportó a Puerto Plata en Alerta Roja, entre los más afectados, con daños severos a la infraestructura vial, personas movilizadas, viviendas afectadas y destruidas, servicios básicos afectados, pérdidas de medios de vida y daños severos a la agropecuaria; también fue afectado parcialmente un (1) muelle y suspendido el arribo de un crucero (COE María, 2017). 
El Municipio de Puerto Plata ha sido intervenido en los aspectos de Gestión de Riesgos en varias ocasiones. Ya en el año 2015 fue clasificado como uno de los diez municipios más vulnerables de la República Dominicana por el estudio de Selección de Municipios del Proyecto "Fortalecimiento de las Estructuras Organizativo-Funcionales de la Gestión de Riesgo ante Desastres en RD" No. FED/2014/353-229, tomando en cuenta su grado de susceptibilidad.

La provincia de Puerto Plata forma parte de la región del Cibao Norte, está ubicada en la costa, en el sector septentrional de la República Dominicana. Tiene nueve municipios y doce distritos municipales. La capital de la provincia es San Felipe de Puerto Plata, donde se ubica el $49 \%$ de la población de la provincia. Tiene dos distritos municipales, Maimón y Yásica Arriba; se encuentra limitada al norte, por el Océano Atlántico, al sur por el municipio de Altamira, al oeste por los municipios de Imbert y Luperón, y al este por el municipio de Villa de Montellano. Fue fundada por Nicolás de Ovando en los inicios de la colonización hispánica, hacia 1502. Se ubica en las coordenadas geográficas $19^{\circ} 48^{\prime} \mathrm{N}-70^{\circ} 41^{\prime} \mathrm{W}$, ocupando una superficie de $459.71 \mathrm{~km}^{2}$, clima tropical, población de San Felipe de Puerto Plata y sus dos distritos municipales, Yásica Arriba (8,791 habitantes), Maimón (21,795 habitantes) con una población total de 158,756 habitantes según el IX Censo de Población y Vivienda 2010; altura sobre el nivel del mar de 8 metros (ONE, 2010).

Los desastres por fenómenos naturales son muy recurrentes en el Municipio de San Felipe de Puerto Plata, lo que nos motivó a desarrollar un trabajo sobre "Gestión de Riesgos y Sostenibilidad" para el cumplimiento final del Curso "Reducción de Riesgo de Desastres y Desarrollo Local Sostenible" en el Centro Internacional de Formación, CIF, de la Organización Internacional del Trabajo, OIT, de la Naciones Unidas (UN). Junio-diciembre 2016, Turín, Italia.
El trabajo se inició con la participación en un proyecto de Gestión de Riesgos en la República Dominicana; en el que se realizó un diagnóstico de riesgo de los municipios vulnerables partiendo de una lista de 45, para seleccionar los 10 más vulnerables. Para ello se tomaron en consideración análisis previos, estudios y reportes de intervenciones realizadas por diversos organismos nacionales e internacionales, entre los cuales están el Documento País 2014 sobre la Gestión de Riesgos en República Dominicana y el Plan Nacional de Gestión Integral del Riesgo de Desastres. También se usó el sistema de indicadores de Riesgo de Desastre y Gestión de Riesgos del Programa para América Latina y el Caribe del Banco Interamericano de Desarrollo, BID, junto con los Indicadores del Progreso para medir la Reducción del Riesgo de Desastres y la Implementación del Marco de Acción de Hyogo de la Estrategia Internacional para la Reducción de Riesgos de la Oficina de Naciones Unidas para la Reducción del Riego de Desastres, (UNISDR). Este estudio confirmó al municipio de San Felipe de Puerto Plata como uno de los 10 más vulnerables.

En la investigación también se realizaron consultas con actores claves del municipio para conocer la operatividad del Comité Municipal de Prevención, Mitigación y Respuesta (PMR), así como una evaluación del riesgo basado en estudios previamente realizados, que evidenció la situación peligrosa en que se encuentran los diversos grupos sociales. De tal manera, el estudio debió servir para promover acciones a nivel comunitario y local.

Como resultados de la investigación para el desarrollo del trabajo se pueden mencionar los siguientes hallazgos, como las causas que contribuyen a generar desastres en el municipio de San Felipe de Puerto Plata:

Las características físico-geográficas del municipio de San Felipe de Puerto Plata, y los 
factores antrópicos presentes en ella, lo exponen a diferentes amenazas, con vulnerabilidades que representan riesgos potenciales. Las principales amenazas del municipio son, geológicas sísmicas y asociadas, como los tsunamis; climáticas por ciclones tropicales y asociadas como deslizamientos e inundaciones, y antrópicas, como la contaminación del río San Marcos y del sistema de cañadas que nacen en la Loma Isabel de Torres y cruzan diferentes puntos de la ciudad (Martínez, 2013).

Las vulnerabilidades física, social, ambiental, económica e institucional, no pueden ser peores y son originadas por una alta concentración de personas en comunidades desfavorecidas; los altos niveles de pobreza en barrios periféricos; un alto nivel de analfabetismo en el municipio correspondiente a un 25\% (ONE, 2010); el deficiente servicio de agua potable en gran parte de la población; la existencia limitada de planes de respuesta ante las diferentes amenazas: inundaciones, deslizamientos, huracanes, tsunamis y contaminación; la no existencia de programas ni de iniciativas en el ayuntamiento local para la reducción de riesgos de desastres; la falta de capacitación de los actores locales en estos temas; la falta de Plan de Ordenamiento Territorial; la falta de coordinación en el Comité Municipal de Prevención, Mitigación y Respuestas (PMR); razones de institucionalidad, política y legal por permisibilidad de las Autoridades Municipales de asentamientos de familias en área que son vulnerables a las amenazas naturales; razones sociales y económicas como la falta de educación y conciencia de la ciudadanía para disponer adecuadamente los desechos sólidos; el vertido de desechos industriales y razones ambientales (Giraldo, 2013).

\section{Objetivos de Desarrollo Sostenible}

Representantes de la mayoría de los países del mundo, reunidos en las Naciones Unidas el
25 de septiembre de 2015, adoptaron conjuntamente 17 Objetivos de Desarrollo Sostenible, centrados en erradicar la pobreza, proteger el planeta y asegurar la prosperidad para todas las personas. Estos objetivos tienen 169 metas y 231 indicadores para lograr el futuro que queremos, dando paso a la Agenda 2030 para el Desarrollo Sostenible, mejor conocida como Agenda 2030. La República Dominicana está comprometida con el cumplimiento de estos objetivos.

\section{Los ODS (ONU, 2015) son:}

Objetivo 1: Poner fin a la pobreza en todas sus formas en todo el mundo.

Objetivo 2: Poner fin al hambre, lograr la seguridad alimentaria y la mejora de la nutrición y promover la agricultura sostenible.

Objetivo 3: Garantizar una vida sana y promover el bienestar en todas las edades.

Objetivo 4: Garantizar una educación inclusiva, equitativa y de calidad y promover oportunidades de aprendizaje durante toda la vida para todos.

Objetivo 5: Lograr la igualdad entre los géneros y empoderar a todas las mujeres y las niñas.

Objetivo 6: Garantizar la disponibilidad de agua y su gestión sostenible y el saneamiento para todos.

Objetivo 7: Garantizar el acceso a una energía asequible, segura, sostenible y moderna para todos.

Objetivo 8: Promover el crecimiento económico sostenido, inclusivo y sostenido, el empleo pleno y productivo y el trabajo decente para todos. 
Objetivo 9: Construir infraestructuras resilientes, promover la industrialización inclusiva y sostenible y fomentar la innovación.

Objetivo 10: Reducir la desigualdad en y entre los países.

Objetivo 11: Lograr que las ciudades y los asentamientos humanos sean inclusivos, seguros, resilientes y sostenibles.

Objetivo 12: Garantizar modalidades de consumo y producción sostenibles.

Objetivo 13: Adoptar medidas urgentes para combatir el cambio climático y sus efectos.

Objetivo 14: Conservar y utilizar en forma sostenible los océanos, los mares y los recursos marinos para el desarrollo sostenible.

Objetivo 15: Proteger, restablecer y promover el uso sostenible de los ecosistemas terrestres, gestionar los bosques de forma sostenible, luchar contra la desertificación, detener e invertir la degradación de las tierras y poner freno a la pérdida de la diversidad biológica.

Objetivo 16: Promover sociedades pacíficas e inclusivas para el desarrollo sostenible, facilitar el acceso a la justicia para todos y crear instituciones eficaces, responsables e inclusivas a todos los niveles.

Objetivo 17: Fortalecer los medios de ejecución y revitalizar la Alianza Mundial para el Desarrollo Sostenible.

Acuerdo Marco para la Reducción de Desastres 2015-2030 de Sendai.
La Gestión de Riesgos a nivel mundial es liderada por "El Marco de Sendai para la Reducción del Riesgo de Desastres 2015-2030", el cual se adoptó en la tercera Conferencia Mundial de las Naciones Unidas celebrada en Sendai (Japón) el 18 de marzo de 2015 (Naciones Unidas, 2015).

El Marco de Sendai es el instrumento sucesor del Marco de Acción de Hyogo para 20052015: su principio es el aumento de la resiliencia de las naciones y las comunidades ante los desastres. Sendai tiene como objetivo lograr la reducción sustancial del riesgo de desastres y de las pérdidas ocasionadas tanto en vidas, medios de subsistencia y salud como en bienes económicos, físicos, sociales, culturales y ambientales de las personas, las empresas, las comunidades y los países.

Para alcanzar el resultado anterior es vital, según Sendai, trabajar para prevenir la aparición de nuevos riesgos de desastres y reducir los existentes implementando medidas integradas e inclusivas de índole económica, estructural, jurídica, social, sanitaria, cultural, educativa, ambiental, tecnológica, política e institucional que prevengan y reduzcan el grado de exposición a las amenazas y la vulnerabilidad a los desastres, aumenten la preparación para la respuesta y la recuperación y refuercen de ese modo la resiliencia.

En el Marco de Sendai se mencionan por primera vez siete metas que incluyen: reducción sustancial de la mortalidad mundial en desastres, reducción sustancial del número de personas afectadas, reducción de las pérdidas económicas en relación al PIB mundial, reducción sustancial de los daños del desastre a la infraestructura y la interrupción de los servicios básicos, incluidos los servicios de salud y educación, aumento en el número de países con estrategias nacionales y locales para la reducción 
del riesgo de desastres para el año 2020, una mayor cooperación internacional, un mayor acceso a peligros múltiples, sistemas de alerta temprana e información del riesgo de desastre y evacuaciones.

Sendai, basada en la experiencia de Hyogo recomienda a los Estados adoptar medidas específicas en todos los planos: local, nacional, regional y mundial, y trabajar en cuatro prioridades que son:

\section{1: Comprender el riesgo de desastres.}

2: Fortalecer la gobernanza del riesgo de desastres para gestionar dicho riesgo.

3: Invertir en la reducción del riesgo de desastres para la resiliencia.

4: Aumentar la preparación para casos de desastre a fin de dar una respuesta eficaz y para "reconstruir mejor" en los ámbitos de la recuperación, la rehabilitación y la reconstrucción.

Como se muestra, la República Dominicana tiene compromisos internacionales que cumplir con El Marco de Sendai para la Reducción del Riesgo de Desastres 2015-2030 y los Objetivos de Desarrollo Sostenible. Por cuanto es una responsabilidad del Estado Dominicano promover a nivel nacional y local estos objetivos y metas.

\section{Conclusión}

Debido a las diferentes intervenciones en la gestión de riesgo en el Municipio de San Felipe de Puerto Plata y a los repetidos efectos negativos asociados a los eventos naturales y actividades humanas, se llega a la conclusión, entre otras cosas, de que existe insuficiencia de entendimiento y sensibilidad de los actores cla- ve del Municipio de San Felipe de Puerto Plata respecto al trabajo coordinado de la gestión de riesgos a desastres naturales y antrópicos. Los daños materiales, el impacto a ecosistemas y las pérdidas de vidas humanas recurrentes por las inundaciones asociadas a las precipitaciones de los meses de noviembre-diciembre de 2016 y las ocasionadas por los Huracanes Irma y María en el 2017; así lo demuestran.

Visto lo anterior, se recomienda inicialmente apoyar el fortalecimiento de los principales actores del Municipio de San Felipe de Puerto Plata de la República Dominicana en el trabajo coordinado de la gestión y reducción de los riesgos a desastres y la adaptación al cambio climático con los siguientes propósitos u objetivos:

1. Motivar a los actores clave del municipio al trabajo coordinado de la gestión y reducción de los riesgos.

2. Promover el conocimiento y la capacitación entre los actores clave del Municipio de San Felipe de Puerto Plata, sobre los riesgos naturales y antrópicos identificados en su territorio.

3. Motivar a las autoridades municipales a incorporar la gestión de riesgos y el cambio climático en sus planes de desarrollo municipal y comunitario de una manera sostenible.

Las líneas de acción para lograr estos objetivos pueden ser:

Definir espacios de coordinación entre todos los actores relacionados con la gestión y reducción de riesgos a desastres.

Elaborar un diagnóstico participativo de riesgos a desastres (naturales y antrópicos) de los dos Distritos Municipales (DM), Maimón y Yásica Arriba del municipio de San Felipe de Puerto Plata. 
Lograr que los actores clave del municipio identifiquen y reconozcan los riesgos naturales y antrópicos presentes en su territorio.

Establecer centros de documentación e información sobre gestión de riesgos de desastres, para consulta de los actores clave y la comunidad en general.

Desarrollar un plan de capacitación comunitaria en gestión integral de riesgos a desastres.

Preparar y realizar un taller municipal sobre gestión de riesgo y cambio climático en los Planes de Desarrollo Municipal en la Provincia de San Felipe de Puerto Plata.

Al mismo tiempo es recomendable desarrollar otros proyectos a nivel local, del gobierno central y organismos internacionales tomando en cuenta el Marco de Sendai, los Objetivos de Desarrollo Sostenible y la Estrategia Nacional de Desarrollo para lograr un desarrollo sostenible (Suriel, 2016).

Los proyectos a desarrollarse dentro del marco del aumento de la resiliencia y de la gestión de riesgos a desastres, ayudarán a cumplir con los objetivos de Desarrollo Sostenible de Naciones Unidas mencionados previamente.

Los trabajos a ejecutarse con estos lineamientos, además de aumentar la sostenibilidad medio ambiental para lograr el futuro que queremos y la reducción de riesgos a desastres; contribuirán con los objetivos y se reflejarán en los indicadores de ODS y con las metas e indicadores del Marco de Sendai para el aumento de la resiliencia de la nación y las comunidades ante los desastres. Específicamente en el caso del Municipio de San Felipe de Puerto Plata; estas medidas son de gran urgencia para que la región sea menos vulnerable y tenga mayores posibilidades de desarrollo sostenible.

\section{Referencias}

Centro de Operaciones de Emergencia COE (2017). Informe Final por Vaguada $8 / 12 / 2016$. Santo Domingo, República Dominicana.

Centro de Operaciones de Emergencia COE (2017). Informe Final Huracán Irma 15/09/2017. Santo Domingo, República Dominicana.

Centro de Operaciones de Emergencia COE (2017). Informe Final Huracán María 02/10/2017. Santo Domingo, República Dominicana.

Dirección General de Ordenamiento y Desarrollo Territorial -DGODT. (2012). Amenazas y Riesgos Naturales en República Dominicana - Compendio de Mapas. Programa de Prevención de Desastres y Gestión de Riesgos. Santo Domingo.

Cocco Quezada, A. (s.f.). Temporada de Huracanes en la República Dominicana. Recuperado de http://www.acqweather.com/HURACANES.htm

Dirección General de Ordenamiento y Desarrollo Territorial -DGODT. (2013). Plan Municipal de Gestión de Riesgos San Felipe de Puerto Plata de la Dirección General de Ordenamiento y Desarrollo Territorial (DGODT), a través del Programa de Prevención de Desastres y Gestión de Riesgos (1708/ OC-DR). Financiamiento del Banco Interamericano de Desarrollo (BID). Santo Domingo.

Gaceta Oficial. Ley 147-02. (2002) Sistema Nacional de Prevención, Mitigación y Respuesta. 
Giraldo, M. (2013). Propuestas integrales y estrategia para la reducción de riesgos. Programa de Prevención de Desastres y Gestión de Riesgos. DGODT - BID. Santo Domingo.

Martínez, I. (2013). Caracterización Amenazas Naturales por Deslizamientos e Inundación. Municipio San Felipe de Puerto. Programa de Prevención de Desastres y Gestión de Riesgos. DGODT - BID. Santo Domingo.

Naciones Unidas. (2015). Marco de Sendai para la Reducción del Riesgo de Desastres 2015-2030. Oficina de las Naciones Unidas para la Reducción del Riesgo de Desastres (UNISDR). Recuperado de www.unisdr.org.

Oficina Nacional de Estadísticas (ONE). (2010). IX CENSO NACIONAL DE POBLACIÓN Y VIVIENDA. Santo Domingo, República Dominicana.

\section{PNUD/ UNESCO/ OIM/ Comisión Europea/} ANESVAD. (2012). Mapa de inundación, rutas de evacuación y zonas seguras en caso de tsunami. Ciudad de Puerto Plata.
Programa DELNET - Organización Internacional del Trabajo-OIT. (2014). El riesgo de desastres: origen, evaluación, reducción y prevención en el marco del desarrollo local sostenible.

Programa DELNET - Organización Internacional del Trabajo-OIT. (2016). Curso: Reducción Del Riesgo De Desastres y Desarrollo Local Sostenible, Programa Delnet CIF/OIT. (2016). Materiales de la Unidad 1 a la 6; Guía, Marco Teórico y Glosario.

Suriel, R. (2016). Fortalecimiento de capacidades de los actores clave del municipio de San Felipe de Puerto Plata al trabajo coordinado de la Gestión de Riesgos de Desastres. Turín, Italia: Programa DELNET - Organización Internacional del Trabajo-OIT.

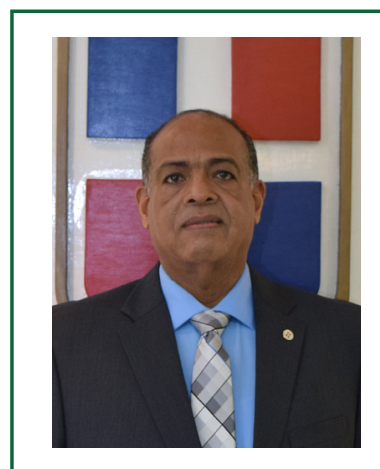

\section{Juan Roberto Suriel Guzmán}

Bore nus rerum et veri ut intionsequi officil lantibus, quis es idi rem aliqui ium sunt. Voluptiae sunt hari te quisi dolorerum earions erepedi assi corio doluptassi consequ amendita is quia nonsed quosa quis enis ped maio qui odi alit facim excesedi volore, officipsusae maiorero doluptibus mil miliatem cor aut eium ut laccumque cum fugitiatur? Soluptas maximus, cum quam, comnimu sdandel essunt maximporerci beatur? As magnimintum sequatem rehendelent harchil int am, velitium voluptat. Volupta temquam, sam nulland itatquam, eumenet quod et, sitionsequat minihitio optatiu sandenis elendant ernatur andae. Itae ne sum et erunt, se num elit que comnit, sequae. Ipitatatis aut re volorum quidus aut ulpa dolor as et laci optatio. Ut ulpa quasper spicidu stiur? Ectem ut que laut omnihit estrum autenih illabor aliquas eatium, il eaquist 